\title{
Supporting physicians in the management of metabolic alterations in adult kidney transplant recipients: a comment on the joint position statement of the Italian Society of Nephrology (SIN), the Italian Society for Organ Transplantation (SITO) and the Italian Diabetes Society (SID)
}

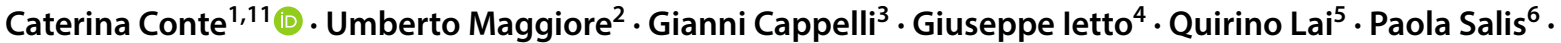 \\ Piero Marchetti ${ }^{7} \cdot$ Lorenzo Piemonti $^{8,9} \cdot$ Antonio Secchi $^{1,9} \cdot$ Enzo Capocasale $^{10} \cdot$ Rossana Caldara $^{1}$
}

Published online: 2 September 2020

(c) Italian Society of Nephrology 2020

Umberto Maggiore, Gianni Cappelli, Giuseppe Ietto, Quirino Lai, Paola Salis, Antonio Secchi, Enzo Capocasale, Rossana Caldara: On behalf of The Joint Committee of the Italian Societies of Nephrology and Organ Transplantation (Collegio SIN-SITO) for kidney and pancreas transplantation.

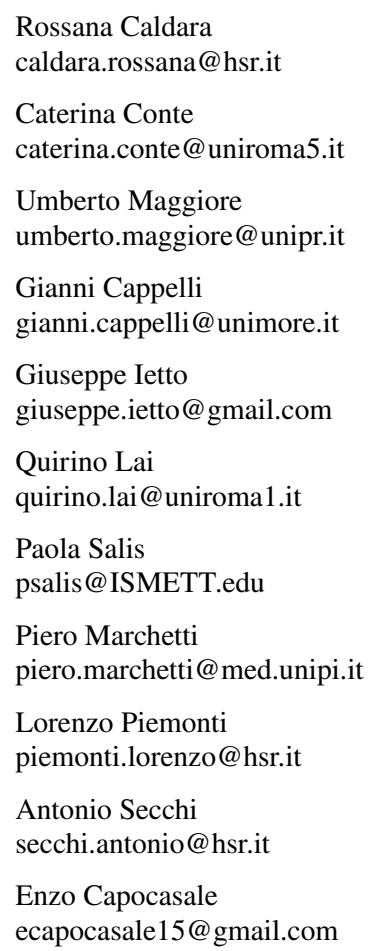

1 IRCCS San Raffaele Hospital, Milan, Italy

2 Dipartimento di Medicina e Chirurgia, University Hospital of Parma, Parma, Italy

3 University of Modena and Reggio Emilia, Azienda Ospedaliero, Universitaria Policlinico, Modena, Italy

4 Ospedale di Circolo e Fondazione Macchi, University of Insubria, Varese, Italy

5 Hepato-Biliary Surgery and Organ Transplantation Unit, Sapienza University of Rome, Umberto I Polyclinic of Rome, Rome, Italy

6 IRCCS ISMETT (Istituto Mediterraneo per I Trapianti e Terapie ad alta Specializzazione), Palermo, Italy

7 Dipartimento di Medicina Clinica e Sperimentale, Università di Pisa, Pisa, Italy

8 Università Vita-Salute San Raffaele, Milan, Italy

9 Diabetes Research Institute, IRCCS San Raffaele Scientific Institute, Milan, Italy

10 Parma University Hospital, Parma, Italy

11 Present Address: Department of Human Sciences and Promotion of the Quality of Life, San Raffaele Roma Open University, Rome, Italy 


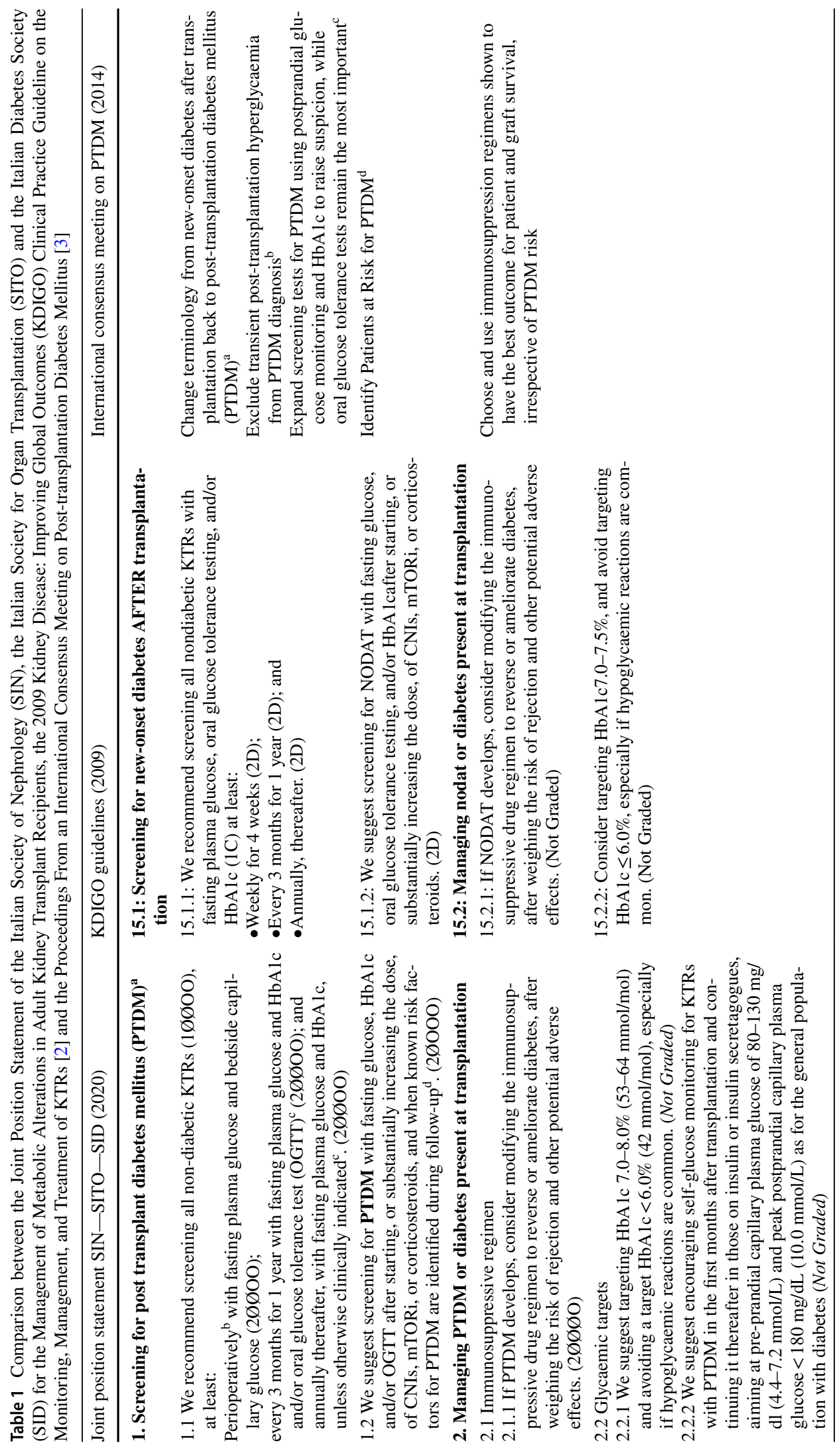




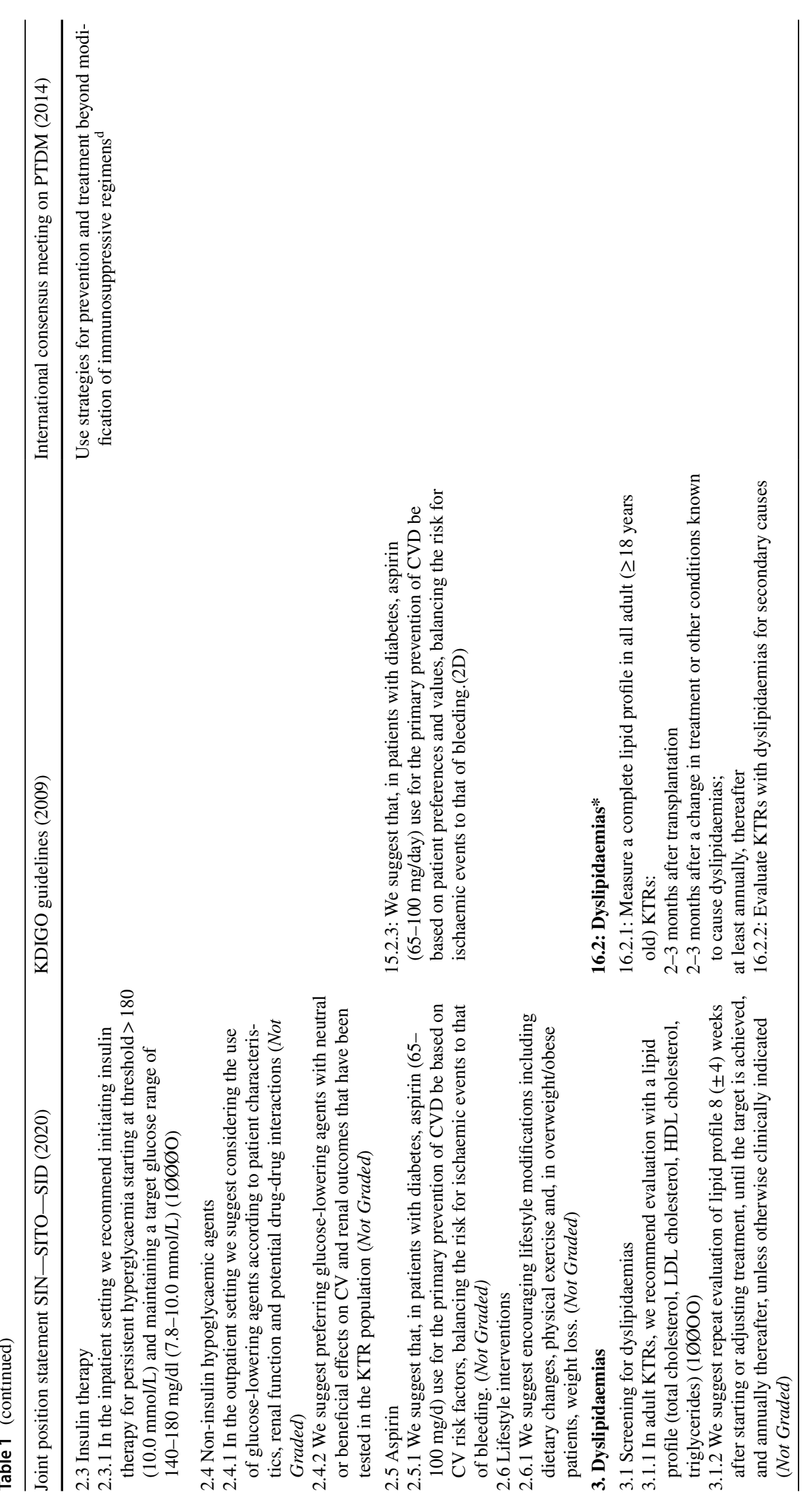




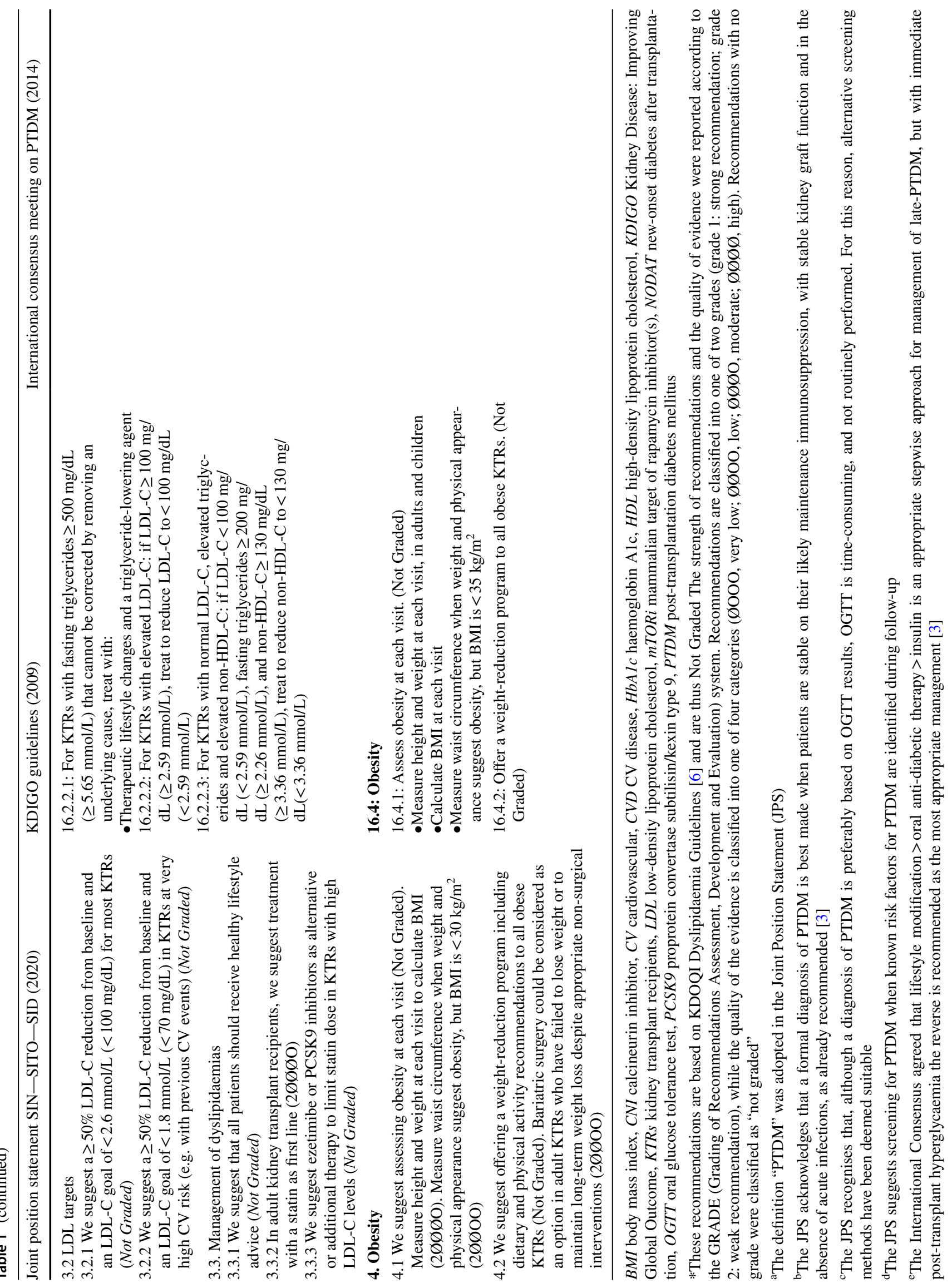


Post-transplant diabetes mellitus, dyslipidaemias and overweight/obesity are extremely common among kidney transplant recipients (KTRs) and may undermine graft and recipient outcomes. With the joint position statement on the management of metabolic alterations in adult KTRs recently issued by the Italian Society of Nephrology, the Italian Society for Organ Transplantation and the Italian Diabetes Society [1], we sought to address the prevention, diagnosis and treatment of metabolic alterations in KTRs in order to support clinical decisions of professionals involved in the management of these patients. The joint position statement was meant to be an update of the 2009 Kidney Disease: Improving Global Outcomes (KDIGO) clinical practice guideline on the monitoring, management, and treatment of KTRs [2] and is based on the evidence published since then. When no new evidence was found, indications were based on expert opinion and relevant guidelines for the nontransplant population. In this commentary addressed to an audience of clinical nephrologists, we review the new statements and compare them with the previous recommendations (Table 1) [2].

1. Screening for post-transplant diabetes mellitus Posttransplant diabetes mellitus had been previously addressed by the KDIGO guidelines and by a consensus of international experts [3]. Although postoperative hyperglycaemia may be predictive of subsequent alterations of glucose metabolism and should prompt close monitoring during follow-up, a formal diagnosis of post-transplant diabetes mellitus is best made when patients are stable on maintenance immunosuppression, with stable kidney graft function and in the absence of acute infections [3]. As compared with previous guidelines [2], the joint position statement makes a distinction between the screening method (measurement of fasting plasma glucose, bedside capillary glucose, HbA1c or the oral glucose tolerance test [OGTT]) depending on the timing of assessment (perioperatively, in the first year after kidney transplantation or thereafter). Bedside capillary glucose is introduced as one of the suggested methods for the perioperative period, as it may be more sensitive than other tests in detecting patients at risk for post-transplant diabetes. The joint position statement confirms the need for screening for post-transplant diabetes after modifying the immunosuppressive drug regimen, and suggests screening when known risk factors are identified.

2. Managing post-transplant diabetes mellitus or diabetes present at transplantation Similar to previous guidelines [2], the joint position statement suggests considering modification of the immunosuppressive drug regimen. While little evidence was available to grade this statement when the KDIGO guidelines were issued, few studies have been published showing that early steroid withdrawal and switching from tacrolimus to cyclosporine (CsA) or from a calcineurin inhibitor-based immunosuppressive regimen to a mammalian target of rapamycin inhibitor (mTORi)-based immunosuppressive regimen might have some benefit on glucose metabolism, although more studies are needed to address these strategies. Data are also needed to assess the effects of reducing the dose of tacrolimus, CsA or corticosteroids; replacing tacrolimus or CsA with mycophenolate mofetil or azathioprine; reducing the dose or discontinuing a mTORi. Importantly, the choice of modifying the immunosuppressive regimen should always be balanced against the risk of rejection and other potential adverse effects [3].

For KTRs with pre-existing diabetes or post-transplant diabetes mellitus, a less stringent glycaemic target $(\mathrm{HbA} 1 \mathrm{c} 7-8 \%$ or $53-64 \mathrm{mmol} / \mathrm{mol})$ is proposed, as compared with previous guidelines [2], based on a large retrospective study suggesting that hypoglycaemia is particularly detrimental to KTRs, who are at increased cardiovascular risk as compared with the general population. Self-glucose monitoring should be encouraged. In the absence of studies specifically addressing this point, the same capillary glucose targets as for the general population with diabetes are suggested.

The joint position statement also addresses the pharmacological management of diabetes, in both the inpatient and outpatient setting, more in depth than previously done [3]. Insulin therapy is strongly recommended for persistent hyperglycaemia in the inpatient setting, and glycaemic targets are proposed to reduce the risk of adverse outcomes or subsequent post-transplant diabetes mellitus. There was not sufficient evidence to guide recommendations on the choice of glucose-lowering agents in the outpatient setting, although the advent of novel drugs (i.e. glucagon-like peptide 1 receptor agonists [GLP-1RA] and sodium-glucose cotransporter-2 inhibitors [SGLT-2i]) has boosted research in this field, and more evidence will likely become available soon. It was deemed reasonable to consider the choice of glucoselowering agents according to patient characteristics, renal function and potential drug-drug interactions, preferring those with neutral or beneficial effects on cardiovascualr and renal outcomes and that had already been tested in KTRs. No new studies were available on aspirin use for primary prevention in KTRs. As previously suggested [2], the use of aspirin can be considered for the primary prevention of cardiovascular disease based on risk factors, after balancing the risk for ischaemic events to that of bleeding. Further studies are needed since, in KTRs requiring urgent graft biopsy, aspirin may have 
the additional disadvantage of increasing the risk of bleeding and/or of delaying the time of biopsy.

Data on lifestyle modifications on prevention and management of metabolic alterations in KTRs were and still are too sparse to support firm recommendations [2, 3]. The joint position statement suggests encouraging lifestyle modifications including dietary changes, physical exercise and, in overweight/obese patients, weight loss. This suggestion was not graded, due to the paucity and low quality of the available evidence. Very recently, the results of a randomised controlled trial were published, suggesting that a 6-month active lifestyle intervention led by renal dieticians did not improve glucose metabolism as compared with passive lifestyle advice after kidney transplantation [4]. These findings should be taken with caution and not discourage physicians from providing lifestyle recommendations, as (1) both the active and passive interventions appeared to improve glucose metabolism to some extent; (2) the active intervention was associated with greater weight and fat mass loss, as well as with a trend towards lower incidence of post-transplant diabetes mellitus. Lack of precise characterisation of baseline glucose metabolism (i.e. normal, impaired fasting glucose or impaired glucose tolerance), different degrees of renal function and the wide timewindow following kidney transplantation (3-24 months) and body mass index range might help explain the lack of significant differences between the two interventions. Future studies should address these aspects and include patients at risk for developing post-transplant diabetes mellitus.

3. Dyslipidaemias Studies specifically addressing the need for dyslipidaemia screening in adult KTRs are lacking. The joint position statement confirms that initial evaluation of the lipid profile is strongly recommended, as it is non-invasive, inexpensive, and allows determining the type and severity of dyslipidaemia [5, 6]. Similar to previous guidelines, repeat evaluation of lipid profile is suggested after starting or adjusting treatment, until the target is achieved and annually thereafter, unless otherwise clinically indicated. While an LDL goal of $<100 \mathrm{mg} / \mathrm{dL}$ ( $<2.6 \mathrm{mmol} / \mathrm{L})$ was previously recommended for all KTRs [1], the joint position statement suggests achieving at least $\mathrm{a} \geq 50 \%$ LDL-C reduction from baseline in all KTRs, but sets different LDL goals based on the cardiovascular risk profile, suggesting that a lower goal of $<70 \mathrm{mg} / \mathrm{dL}[<1.8 \mathrm{mmol} / \mathrm{L}]$ for KTRs at very high cardiovascular risk. These goals appear to be realistic and of potential benefit. While no specific indications on the pharmacological management of dyslipidaemias were provided previously, the joint position statement suggests statins as first-line lipid-lowering treatment, as there is evidence that these agents might reduce cardiovascular events in KTRs. Use of ezetimibe or PCSK9 inhibitors may be considered as alternative or additional therapy to limit statin dose, but studies are needed to confirm the efficacy and safety of these agents in KTRs.

4. Obesity As in previous guidelines, assessment of obesity is recommended at each visit, although the strength of recommendation is weak due to the limited number of studies addressing this point. As waist circumference may predict long-term survival better than BMI in KTRs, measuring it is suggested when weight and physical appearance suggest obesity, but BMI is lower than the threshold for diagnosing obesity $\left(<30 \mathrm{~kg} / \mathrm{m}^{2}\right)$. At the time the KDIGO guidelines were published, only anecdotal evidence was available to support the use of bariatric surgery in the management of obese KTRs. Several reports have been published since then, although of low quality, and suggest that bariatric surgery in KTRs is effective for weight loss and associated with low rates of complications and mortality. Given the potential benefits of bariatric surgery on graft function, survival, and obesity-related co-morbidities, further studies are needed to better define indications, type of procedure and weight loss targets in the management of obese KTRs.

With the present joint position statement, we sought to provide practical indications to support clinicians in the management of KTRs. The choice of treatment, however, remains individual and depends on several factors such as the expected risk/benefit ratio for each patient, his/her preferences, and the availability of healthcare resources. A strength of this position statement is that the recommendations stemmed from the joint effort of experts in diabetology and metabolism, nephrology, and kidney transplantation, but several gaps still need to be filled to guide decisions. The efficacy and safety of glucose- and lipid-lowering drugs that have recently become available, the role of bariatric surgery and the identification of subpopulations of KTRs who would benefit the most from lifestyle interventions are just some of the important issues that should be urgently addressed.

Funding $\mathrm{CC}$ is supported by the European Foundation for the Study of Diabetes Mentorship Programme 2019.

\section{Compliance with ethical standards}

Conflict of interest The authors declare that they have no conflict of interest.

Ethical approval Not applicable. 
Informed consent Not applicable.

\section{References}

1. Conte C, Maggiore U, Cappelli G, Ietto G, Lai Q, Salis P, Marchetti P, Piemonti L, Secchi A, Capocasale E, Caldara R (2020) Management of metabolic alterations in adult kidney transplant recipients: a joint position statement of the Italian Society of Nephrology (SIN), the Italian Society for Organ Transplantation (SITO) and the Italian Diabetes Society (SID). Nutr Metab Cardiovasc Dis 2020;30(9):1427-1441. https://doi.org/10.1016/j. numecd.2020.05.004

2. Kidney Disease: Improving Global Outcomes Transplant Work G (2009) KDIGO clinical practice guideline for the care of kidney transplant recipients. Am J Transpl 9(Suppl 3):S1-155. https:// doi.org/10.1111/j.1600-6143.2009.02834.X

3. Sharif A, Hecking M, de Vries AP, Porrini E, Hornum M, RasoulRockenschaub S, Berlakovich G, Krebs M, Kautzky-Willer A, Schernthaner G, Marchetti P, Pacini G, Ojo A, Takahara S, Larsen JL, Budde K, Eller K, Pascual J, Jardine A, Bakker SJ, Valderhaug TG, Jenssen TG, Cohney S, Saemann MD (2014) Proceedings from an international consensus meeting on posttransplantation diabetes mellitus: recommendations and future directions. Am J Transpl 14(9):1992-2000. https://doi.org/10.1111/ajt.12850

4. Kuningas K, Driscoll J, Mair R, Smith H, Dutton M, Day E, Sharif AA (2020) Comparing glycaemic benefits of active versus passive lifestyle intervention in kidney allograft recipients: a randomized controlled trial. Transplantation 104(7):1491-1499. https://doi. org/10.1097/TP.0000000000002969

5. Tonelli M, Wanner C, Kidney Disease: Improving Global Outcomes Lipid Guideline Development Work Group M ( 2014) Lipid management in chronic kidney disease: synopsis of the Kidney Disease: improving Global outcomes 2013 clinical practice guideline. Ann Intern Med 160(3):182. https://doi.org/10.7326/M13-2453

6. Wanner C, Tonelli M, Kidney Disease: Improving Global Outcomes Lipid Guideline Development Work Group M (2014) KDIGO clinical practice guideline for lipid management in CKD: summary of recommendation statements and clinical approach to the patient. Kidney Int 85(6):1303-1309. https://doi.org/10.1038/ki.2014.31

Publisher's Note Springer Nature remains neutral with regard to jurisdictional claims in published maps and institutional affiliations. 